\title{
Projetos PET-Saúde e Educando para a Saúde: Construindo Saberes e Práticas
}

\author{
The Educational Program for Health Work \\ and the Educating for Health Project: \\ Building Knowledge and Practices
}

\author{
Pâmela Leites de Souzal \\ Celeste dos Santos Pereira ${ }^{I}$ \\ Maria Laura Silveira Nogueira II \\ Denise Bermudez Pereirall \\ Giolana Mascarenhas da Cunha ${ }^{I}$ \\ Fabiana de Oliveira Möler
}

\section{PALAVRAS-CHAVE \\ - Saúde Escolar; \\ - Educação em Saúde; \\ - Ação Intersetorial; \\ - Atenção Primária à Saúde.}

Recebido em: 20/09/2010

Aprovado em: 19/11/2010

REVISTA BRASILEIRA DE EDUCAÇÃO MÉDICA
${ }^{I}$ Universidade Federal de Pelotas, Pelotas, RS, Brasil.

II Secretaria Municipal de Saúde de Pelotas, Pelotas, RS, Brasil.

\section{RESUMO}

Buscando alcançar ações intersetoriais efetivas e inovadoras, o projeto Educando para a Saúde, vem envolvendo as escolas, academia e serviços de saúde locais no desafio de construir uma sólida relação entre saúde-escola. O presente relato de experiência socializa a trajetória do projeto Educando para a Saúde, uma parceria entre a Equipe de Saúde da Família (ESF) de um bairro de Pelotas - RS, a comunidade docente de duas escolas do território e acadêmicos do Programa de Educação pelo Trabalho para a Saúde - PET-Saúde. O objetivo é refletir o papel da ESF e dos acadêmicos do Projeto PET-Saúde na integração saúde-educação. Os temas discutidos são relacionados à educação em sexualidade, direitos sexuais e reprodutivos com ênfase nas DSTs e métodos contraceptivos, prevenção, promoção e recuperação da saúde. A participação do acadêmico nas atividades de integração saúde-educação veio a acrescentar ideias, valores e conceitos, possibilitando a (re)construção das relações interpessoais, promovendo a reciclagem do processo de trabalho. Pelos laços multiprofissionais e interdisciplinares, essa parceria consiste em aprendizado mútuo, cujos frutos refletem diretamente na melhoria da assistência prestada à comunidade.

Seeking to achieve effective and innovative inter-sector action, the Educational Program for Health Work has been involving schools, academia, and local health services in the challenge of building a solid relationship between health and schools. The current case report shares the experience with the Educating for Health project, a partnership between the Family Health Team (FHT) from a neighborhood in Pelotas, Rio Grande do Sul State, teachers from two local schools, and students from the Educational Program for Health Work (PET-Saúde). The aim was to reflect on the roles of the FHT and students from the PET-Saúde project in the integration between health and education. The themes discussed in the process relate to sex education, sexual and reproductive rights with an emphasis on sexually transmitted diseases and contraception, prevention, health promotion, and recovery/rehabilitation. Students' participation added ideas, values, and concepts, fostering the (re)construction of interpersonal relations and promoting a recycling of the work process. Based on multi-professional and interdisciplinary linkages, this partnership became a mutual learning process, the results of which are reflected directly by improvement in healthcare for the community. 


\section{INTRODUÇÃO}

O Brasil conta com mais de 54 milhões de cidadãos e cidadãs na faixa de 10 a 24 anos de idade, o que representa $30,3 \%$ da população. Os sistemas de ensino abrigam aproximadamente $62 \%$ dessa população. ${ }^{1}$ Além de sua grande relevância para o País, essas parcelas expressivas da população estão expostas a riscos e relações de vulnerabilidade de caráter estrutural a serem superados de forma premente. ${ }^{1}$ Nesse contexto, o ambiente escolar surge como espaço facilitador para a prevenção de riscos e promoção da saúde de crianças e jovens, além da possibilidade de transformar o quadro de fragilidade social em que muitos deles vivem atualmente. ${ }^{2}$ Trata-se de um espaço institucional privilegiado para a convivência social, cidadania e para o estabelecimento de relações intersubjetivas favoráveis à promoção da saúde e à construção de uma resposta aos desafios colocados para a sociedade. ${ }^{3}$

Dentro da área da saúde, as Equipes de Saúde da Família (ESF) são protagonistas corresponsáveis pela saúde e qualidade de vida das populações onde estão inseridas, podendo atuar como desencadeadores de iniciativas de promoção da saúde escolar em todas as localidades e espaços, principalmente naqueles em que essa questão ainda é incipiente. ${ }^{3} \mathrm{O}$ território é espaço de produção da vida, de valores, crenças e, portanto, da saúde. Dessa forma, o conceito de território é central para a abordagem dos princípios da Atenção Básica, a partir da Equipe de Saúde da Família e seu papel junto à comunidade. É nesse espaço geográfico que contempla unidades escolares e os sujeitos de sua comunidade que as equipes de saúde podem construir e fortalecer a articulação com a população escolar. ${ }^{3}$

Essa parceria deve se estruturar e solidificar levando-se em consideração os limites e as inúmeras possibilidades de atuação, de forma objetiva e dinâmica, estabelecendo um diálogo entre saúde e educação com uma abordagem o mais próxima possível da realidade local dos jovens e suas famílias. Trata-se de um desafio que não diz respeito apenas aos profissionais de saúde e de educação, mas aos estudantes, usuários do serviço de saúde, gestores e governantes, além de representações sociais e acadêmicas.

Além disso, quando se planeja uma atividade que mobilize os sujeitos para a mudança de vida e para se tornarem multiplicadores dos conhecimentos adquiridos, é necessário repensar as potencialidades e os limites da educação preventiva. Uma vez que o conhecimento científico, embora seja um direito, não é uma verdade absoluta e permanente que orienta escolhas racionais, nem é suficiente para aumentar a liberdade de decisão das pessoas. ${ }^{4}$ Não há dúvidas de que as formas de conduzir a vida podem ser beneficiadas pelo saber científico, mas é essencial que ele seja capaz de dialogar com o saber empírico e prático das pessoas e grupos, oferecendo elementos que possam fazer sentido no contexto de vida no qual os sujeitos estejam envolvidos. ${ }^{4}$ Nesse sentido, o conceito de vulnerabilidade social e intersetorialidade como instrumento potencializador são as ferramentas principais para a abertura de novos caminhos a seguir.

O presente relato de experiência socializa a trajetória do projeto Educando para a Saúde, uma parceria entre a Equipe de Saúde da Família de um bairro de Pelotas - RS, a comunidade docente de duas escolas de Ensino Fundamental do território e acadêmicos do Programa de Educação pelo Trabalho para a Saúde - PET-Saúde, a fim de fortalecer iniciativas propostas e repensar estratégias na área. Tem por objetivo refletir o papel da equipe de ESF e dos acadêmicos do projeto PET-Saúde, na integração saúde-educação.

\section{A SAÚDE DO ESCOLAR NO BRASIL}

Para compreender a proposta deste relato, é necessário traçar um breve panorama acerca da saúde do escolar no País, suas conquistas e fragilidades ao longo das décadas. Elementos de saúde e de educação estão presentes durante todo o desenvolvimento humano de maneira substancial, sendo fundamentais na construção biopsicossocial e na formação dos sujeitos sociais e políticos. ${ }^{1}$ Não obstante, a intersetorialidade e a integralidade sempre foram desafios para o Brasil.

Somente em 1986, a partir da Primeira Conferência Internacional sobre Promoção da Saúde, em Ottawa, o conceito de Promoção da Saúde foi introduzido, considerando-a como processo educativo que valoriza o indivíduo, sua cultura e hábitos. ${ }^{5}$ Com a Constituição Federal de 1988, surge a premissa da reorganização dos serviços de saúde, tendo em vista a construção do Sistema Único de Saúde (SUS). Contemplar os princípios do SUS de regionalização, descentralização, acesso universal, integralidade com prioridade para as ações preventivas e de promoção da saúde, além do controle social, implica, também, rever a questão da atenção à saúde da criança e do adolescente, atentando para a marginalização do atendimento realizado pelo setor de saúde até então. ${ }^{6}$

Quase uma década depois, o Ministério da Saúde, por intermédio da Coordenação Nacional de DST/Aids, iniciou trabalho com professores e jovens no âmbito escolar. Foram desenvolvidas atividades de formação de multiplicadores, professores e jovens, a fim de capacitá-los para esse novo olhar à saúde escolar. ${ }^{5}$ A partir de 1999, foram implantados pela Coordenação Nacional de DST/Aids diversos projetos em parceria com estados e municípios, visando a ações nas escolas públicas. A partir desses projetos foram realizadas diversas oficinas e elaborados vários materiais que buscavam a 
prevenção das DSTs/Aids e do uso de drogas entre os jovens escolares. Apesar dos resultados positivos para o setor educativo, encontraram-se fragilidades institucionais na sustentabilidade dessas atividades dentro e fora da escola. ${ }^{5}$

Em 2003, o Ministério da Saúde, por meio da Coordenação Nacional de DST/Aids, e o Ministério da Educação, com apoio da Unesco, Unicef e UNFPA, iniciaram o projeto Saúde e Prevenção nas Escolas (SPE), com o objetivo de reduzir a vulnerabilidade dos jovens, promovendo a saúde sexual e reprodutiva de forma ampla. ${ }^{5}$ Em 2007, o Decreto Presidencial no 6.286 instituiu o Programa Saúde na Escola (PSE), uma política intersetorial - envolvendo os Ministérios da Saúde e da Educação - na perspectiva da prevenção, promoção e atenção integral à saúde de crianças, adolescentes e jovens do ensino público básico. As ações acontecem no ambiente escolar e nas unidades básicas de saúde e são realizadas pelas Equipes de Saúde da Família e profissionais de educação. ${ }^{5} \mathrm{O}$ programa surge para unificar diversas ações que eram executadas pela saúde e pela educação, tais como o Olhar Brasil e o projeto Saúde e Prevenção nas Escolas (SPE), entre outras. ${ }^{2}$

Percebe-se que, a partir dos anos 1950 até o início dos anos 2000, passando pela redemocratização do Brasil e a Constituição, muitas foram as iniciativas e abordagens que pretendiam focalizar o espaço escolar a partir de uma perspectiva sanitária. ${ }^{3}$ Em sua maioria, tais experiências centravam-se no modelo biomédico, através de ações para o controle e tratamento de agravos ou doenças, bem como da garantia de assistência de alta especificidade e tecnologia. ${ }^{1,3}$ Entretanto, hoje se busca outro caminho, o do conceito ampliado de saúde, em que os aspectos biopsicossociais são determinantes na qualidade de vida da população independentemente de sua faixa etária.

\section{PET-SAÚDE: ESTABELECENDO PARCERIAS}

A formação de um profissional de saúde crítico, reflexivo, pró-ativo, preparado para atuar em equipe e no mercado de trabalho exige uma série de experiências de ensino e aprendizagem diferenciadas. O cenário de trabalho no SUS e no mercado estão em transformação, e a Atenção Primária à Saúde (APS) passou a ser essencial para a formação do novo profissional. ${ }^{7}$ Os Ministérios da Saúde e da Educação, entendendo isso como uma necessidade de corroborar com a implantação do SUS, têm promovido várias iniciativas para estimular a aproximação das Universidades e dos serviços de saúde não hospitalares, como nos casos do Pro-Med, do Pró-Saúde e do PET-Saúde, entre outros. ${ }^{7}$ O Programa PET-Saúde, inspirado no Programa de Educação Tutorial - PET, do Ministério da Educação, foi criado em 2008 como uma das ações para forta- lecer a atenção básica em saúde, de acordo com os princípios e as necessidades do SUS. ${ }^{8}$ A educação pelo trabalho, conceito-chave do projeto, é uma das estratégias do Programa Nacional de Reorientação da Formação Profissional em Saúde, o Pró-Saúde, em implementação no País desde 2005. ${ }^{8}$ Entre abril e dezembro de 2009, o Ministério da Saúde concedeu 51.567 bolsas PET-Saúde, um investimento de R 29,5 milhões. Foram selecionados 84 projetos, totalizando 306 grupos que desenvolvem atividades educativas e pesquisas em aproximadamente 820 unidades do Programa Saúde da Família no País. ${ }^{8}$ O PET-Saúde tem como fio condutor a integração ensino, serviço e comunidade, sendo uma parceria entre as secretarias de Gestão do Trabalho e da Educação na Saúde (SGTES) e de Atenção à Saúde (SAS), do Ministério da Saúde juntamente com a Secretaria de Educação Superior (SESU), do Ministério da Educação. ${ }^{8}$ A finalidade é que o aluno transponha os muros da instituição, ampliando e enriquecendo a sua visão sobre a realidade sociopolítica local por meio da Unidade de Saúde da Família em que está inserido.?

Através do perfil do programa e sua estreita relação com a rotina da USF, protagonista do presente estudo, o PET-Saúde somou forças aos projetos e atividades desenvolvidos na Unidade, em especial ao projeto em questão, Educando para a Saúde, cuja experiência será partilhada a seguir.

\section{TRAÇANDO O PROJETO EDUCANDO PARA A SAÚDE}

Diante da importância da educação em saúde como norteadora das ações no âmbito da Estratégia de Saúde da Família, o município de Pelotas, no Rio Grande do Sul, desenvolveu, no ano de 2008, uma capacitação interdisciplinar e intersetorial em que se discutiu o planejamento e a implementação do projeto Saúde e Prevenção nas Escolas - SPE, na cidade. O presente relato, de caráter descritivo, aborda a experiência vivenciada pelos membros da Equipe de Saúde da Família de uma Unidade Básica de Saúde e acadêmicos do projeto PET-Saúde, durante o período de março a agosto de 2010, no bairro Simões Lopes, na cidade de Pelotas, RS. Apresenta as atividades educativas desenvolvidas a partir do projeto Educando para a Saúde, em uma parceria entre a Equipe de Saúde da Família de Simões Lopes, a comunidade docente de duas escolas do bairro e o PET-Saúde. A participação deste último ocorreu de forma gradativa e indissociável das atividades, uma vez que a própria presença do projeto nas unidades prevê a integração entre ensino, serviço e comunidade.

O projeto Educando para a Saúde tem como base e inspiração as normas e diretrizes do programa SPE; seu objetivo é implantar ações integrativas entre saúde e educação, abordando temas relativos à prevenção, promoção e recuperação da 
saúde, com especial ênfase aos direitos reprodutivos e sexuais dos adolescentes.

Os encontros são realizados semanalmente, todas as terças-feiras à tarde e quintas-feiras pela manhã, e direcionados à comunidade escolar local, com faixa etária entre 6 e 19 anos de idade que cursam o Ensino Fundamental. A temática de cada encontro é definida pelos alunos e professores sempre na semana anterior à atividade, a partir de assuntos vistos em aula. Em seguida, é realizado um levantamento das dúvidas e questões relevantes apontadas, as quais serão trabalhadas com a equipe de saúde e membros da equipe docente, sendo que, eventualmente, os pais e familiares são convidados a participar da interação.

A abordagem, a didática e os recursos utilizados para a discussão do tema são definidos conforme as características e realidade da população-alvo. Esse processo leva ainda em consideração aspectos referentes à fase de crescimento e desenvolvimento de cada faixa etária. Os encontros são compostos por oficinas de educação em saúde, debates, bate-papos educativos, dinâmicas de grupo, além de recursos lúdicos, didáticos e ilustrativos.

As ações são multi e interdisciplinares, contando com os profissionais de Medicina e Enfermagem da ESF, equipe de apoio (Nutrição, Serviço Social e Odontologia) e os acadêmicos do PET-Saúde da Universidade Federal de Pelotas. Serão realizadas avaliações semestrais, com análise de resultados para o aproveitamento e planejamento das ações seguintes.

\section{CONSTRUINDO SABERES E PRÁTICAS}

Buscando alcançar ações intersetoriais efetivas e inovadoras, o projeto Educando para a Saúde vem envolvendo as escolas, academia e serviços de saúde locais no desafio de construir uma sólida relação entre saúde-escola, resultando em melhoria no estado biopsicossocial da comunidade escolar, bem como na promoção da qualidade de vida entre os envolvidos.

Os temas discutidos são relacionados à educação em sexualidade, direitos sexuais e reprodutivos, com ênfase nas DSTs e métodos contraceptivos, prevenção, promoção e recuperação da saúde. As ações foram classificadas conforme duas categorias temáticas e relacionam as percepções prévias obtidas desde a concretização do projeto.

\section{Sexualidade, Direitos Sexuais e Reprodutivos}

Perguntas relacionadas à sexualidade, métodos contraceptivos e doenças sexualmente transmissíveis (DSTs) foram as mais solicitadas pelos jovens. Observa-se certa contenção ao exprimir dúvidas a respeito desses temas e expor suas angústias e sentimentos diante do leque de novas experiências que começam a surgir com o amadurecimento. Essa repressão parte não só dos pais, colegas ou professores, mas da sociedade em geral.

De acordo com a Organização Mundial da Saúde, a sexualidade é uma necessidade básica e um componente do ser humano que não pode ser dissociado de outros aspectos da vida. ${ }^{4}$ Sexualidade é muito mais do que o coito e não se limita à presença ou não do orgasmo, é a energia que motiva o encontro para o amor, o contato e a intimidade, implicando em saúde física e mental. ${ }^{4}$ Se a saúde é um direito primordial, a saúde sexual também deve ser considerada um direito humano básico, que envolve o corpo, a história, as relações afetivas e a cultura dos homens. ${ }^{4}$ Apesar disso, ainda há relutância em problematizar esse tema abertamente, como parte da educação de crianças e jovens partindo ainda do ambiente familiar.

A proposta do projeto contribui para este repensar e exerce o papel de conscientização em uma população que cada vez mais apresenta histórico de DSTs/HIV, gestações precoces e frustrações impactantes no âmbito emocional. A idade média de iniciação sexual dos brasileiros situa-se na faixa dos 15 anos de idade, justificando a necessidade de ações de saúde enfáticas direcionadas à população adolescente e jovem. ${ }^{1}$ Os bate-papos educativos constituem a estratégia principal na abordagem desses assuntos, uma vez que se torna ferramenta de debate que, pela informalidade, permite que os alunos, bem como pais e professores, se expressem e se sintam à vontade para expor suas ideias e anseios.

Oficinas com a demonstração dos métodos contraceptivos existentes, permitindo que visualizem cada um deles, assim como a instrução da forma como devem ser utilizados, são recursos especialmente válidos para suscitar o interesse dos estudantes e assumir impacto maior no entendimento de sexo seguro e anticoncepção. Tratar de temas atuais, normalmente estigmatizados e reprimidos anteriormente, possibilita a tomada de consciência acerca da sua existência, desmitificando tabus e contribuindo para a redução das desigualdades sociais e de gênero, do preconceito e da discriminação.

\section{Higiene, Hábitos de Saúde e Agravos Transmissíveis}

Os encontros do projeto abrangem também as classes das primeiras séries do Ensino Fundamental, cujas necessidades a serem supridas se diferenciam em alguns aspectos básicos, referentes ao universo infantil e todo o desconhecimento e ingenuidade inerentes. Ao passo que as questões são mais simples, o interesse e a curiosidade são maiores. Cada assunto tratado, por mais pontual que seja, sempre abrirá um leque de dúvidas e propostas não relacionadas ao tema que invadem as mentes pueris com uma velocidade ímpar. A escola assume durante a 
infância, época decisiva na construção de hábitos e atitudes, importante papel por seu potencial para o desenvolvimento de um trabalho sistematizado e contínuo. Os valores que se expressam na escola em seus diferentes aspectos, geralmente, são apreendidos pelas crianças na sua vivência diária. ${ }^{9}$

Os temas mais trabalhados foram: noções e hábitos de higiene; alimentação saudável; prevenção da transmissão de parasitas, como o piolho e a sarna; gripe H1N1; e sintomatologia e tratamento de algumas doenças aleatórias, conforme colocações do grupo. Recursos lúdicos e atividades educativas foram fundamentais para o desenvolvimento do tema abordado. A inserção no universo infantil aproxima o profissional da criança, facilitando a criação de vínculo e, consequentemente, de confiança no que está sendo compartilhado. Ademais, a interação obtida com as crianças motiva a continuidade do trabalho, uma vez que elas são sinceras nos sentimentos expressos de aprovação ou reprovação, permitindo avaliar as ações realizadas a cada encontro.

\section{PARTICIPAÇÃO DA COMUNIDADE DOCENTE, DISCENTE E FAMILIARES}

A efetiva participação juvenil é, e sempre será, um desafio a ser conquistado a cada encontro, definir estratégias para que se reestruturem os métodos de discussão, estimulando os jovens a auxiliarem nesse processo, é fundamental para que se identifiquem e realmente assimilem e assumam papéis de saúde e cidadania.

O fato de mobilizarmos os próprios alunos no planejamento e definição dos temas a serem tratados aproxima a atividade das reais necessidades dessas crianças e jovens, refletindo as principais carências existentes no currículo das escolas e na relação saúde-população. Exemplos disso são as questões levantadas pelos alunos, que variam desde as mais básicas e casuais às mais complexas e preocupantes. Esses achados chamam atenção para a necessidade de uma boa formação docente, incluindo, desde a graduação, temáticas de saúde, além da devida reciclagem através da educação permanente em saúde.

Os professores são peças-chave nas discussões e também para a continuidade do trabalho. Além disso, os episódios em que os pais e familiares são convidados a participar do debate somam forças para que os objetivos sejam alcançados, uma vez que se tornam multiplicadores, fortalecendo a possibilidade de reprodução de hábitos de vida mais saudáveis e conscientes a partir da família. Afinal, a família constitui a unidade primária de cuidado em saúde, é a entidade que transmite os valores éticos e humanitários regentes de seus membros, na qual se aprofundam laços de solidariedade e respeito.
O interesse, a receptividade e a interação percebidos foram muito válidos e se refletiram na satisfação e participação das crianças, jovens e demais atores do projeto, o que serve de incentivo à melhoria e continuidade do trabalho realizado.

\section{O PROFISSIONAL DE SAÚDE E O ACADÊMICO PET- SAÚDE COMO EDUCADORES POTENCIAIS}

A abordagem de forma dinâmica e mais próxima possível à realidade local busca maior interação entre os alunos, professores e profissionais de saúde, resgatando papéis e vínculos da Unidade Básica de Saúde junto aos jovens e suas famílias. De modo semelhante, as Equipes de Saúde da Família podem contar com o apoio dos profissionais de educação, incorporando, a partir dessa troca, ferramentas pedagógicas e educacionais à sua abordagem de educação em saúde na Unidade de Saúde da Família.

Muitos obstáculos ainda precisam ser transpostos em relação à criação/fortalecimento do vínculo existente entre o escolar e a Unidade Básica de Saúde. O profissional de saúde deve capacitar-se, sensibilizar-se e preparar-se cada vez mais para receber e atender a essa parcela expressiva da população, muitas vezes ignorada. A disponibilização de insumos de prevenção e a redução do estigma e preconceito são temas que deverão ser trabalhados com profissionais dos dois setores. ${ }^{5}$ Ademais, o reconhecimento dos direitos sexuais e reprodutivos dos adolescentes e jovens necessita ser enfrentado por educadores, pais e profissionais de saúde, para que as ações sejam efetivas.

Para o aluno da graduação, empenhar-se em ações intersetoriais de educação em saúde permite um contato valioso com a realidade que encontrará enquanto profissional responsável por uma Unidade Básica de Saúde. Amplia a visão do acadêmico acerca da comunidade como um todo, fortalecendo a crítica reflexiva proposta pela academia, definindo o perfil do profissional que pretende ser. A participação dele nas atividades de integração saúde-educação veio a acrescentar ideias, valores e conceitos ao trabalho com grupos diversos, além de sua identificação e contribuição como membro da equipe de saúde.

Sua presença possibilita, ainda, a (re)construção de relações interpessoais entre o acadêmico e o profissional de saúde, promovendo a reciclagem do processo de trabalho. Os laços com o multiprofissional e o interdisciplinar consiste em aprendizado mútuo, cujas vantagens e frutos refletem diretamente na melhoria da assistência prestada à comunidade.

\section{APONTANDO CAMINHOS}

A abrangência e complexidade dos desafios a serem enfrentados apontam para um novo caminho: o da articulação de políticas para valorizar as contribuições setoriais possíveis e necessárias, assim como a participação da sociedade civil, de 
forma que esta união de capacidades, recursos e responsabilidades possa produzir transformações mais efetivas nas condições geradoras de vulnerabilidade das populações jovens. ${ }^{1}$ As experiências que deram certo, aliadas aos desafios e barreiras existentes, sugerem o desenvolvimento de novas estratégias e investimentos para que as metas dos projetos sejam alcançadas.

Acredita-se que, independentemente da forma e dos métodos de construção dessa relação, o resultado será sempre o mesmo: saúde e educação, desde que haja esse comprometimento. Por fim, o principal desafio situa-se no interesse, na disponibilidade e no compromisso de assumir a saúde escolar, uma vez que muitos são os projetos no papel, mas poucas são as ações realmente desenvolvidas. O educar em saúde, intimamente associado ao formar cidadãos críticos e conscientes, não significa apenas transmitir informação, mas fornecer meios para a construção do conhecimento.

\section{REFERÊNCIAS}

1. Brasil. Ministério da Saúde. Secretaria de Vigilância em Saúde . Programa Nacional de DST e Aids. Diretrizes para implantação do Projeto Saúde e Prevenção nas Escolas. . Brasília: MS; 2008. (Série A. Normas e Manuais Técnicos)

2. Fonseca FF. A escola como espaço facilitador para promoção da saúde e prevenção de riscos. Anais do Encontro Nacional de Ensino de Ciências da Saúde e do Ambiente - Unipli. 2008 mai.15-17, Niterói/RJ, Brasil. 19-23 p.

3. Brasil. Ministério da Saúde. Secretaria de Atenção à Saúde. Departamento de Atenção Básica Saúde na escola. Brasília: MS; 2009. (Série B. Textos Básicos de Saúde) Cadernos de Atenção Básica; n. 24.

4. Brasil. Ministério da Saúde. Secretaria de Vigilância em Saúde. Saúde e prevenção nas escolas: guia para a formação de profissionais de saúde e de educação / Brasília: MS; 2006. (Série A. Normas e Manuais Técnicos).

5. Gomes MRO, Vieira N. . Saúde e Prevenção nas Escolas: Promovendo a Educação em Sexualidade no Brasil. Rev Temp Act em Saúde Col. [periódico na internet]. 2010 [acesso em 12 ago. 2010];2(2):p. 145-7. Disponível em: http:/ /164.41.105.3/ index.php/tempus/article/viewFile/798/786

6. Cyrino EG, Pereira MLT. Reflexões sobre uma proposta de integração saúde-escola: o projeto saúde e educação de Botucatu, São Paulo. Cad Saúde Pública [periódico na internet].1999 [acesso em 12 ago. 2010];15(supl. 2):3944. Disponível em: http://www.scielosp.org/scielo. php?script=sci_arttext\&pid=S0102-311X1999000600005

7. Souza AC, Gama C, Gonçalves MR, Pinto MEB. Experiência interdisciplinar em equipe multiprofissional na graduação em cenário de atenção primária a saúde - PET-Saúde UFCSPA. Anais do $4^{\circ}$ Congresso Multidisciplinar de Saúde Comunitária do Mercosul. In: $4^{\circ}$ Congresso Multidisciplinar de Saúde Comunitária do Mercosul, Gramado-RS, Brasil, 28-30 out., 2009.

8. Brasil. Ministério da Saúde. PET-Saúde: Objetivos, Resultados esperados e Legislação. [on-line]. [acesso em 14 set. 2010] Disponível em: http://portal.saude.gov.br/portal/ saude/profissional

9. Fernandes MH, Rocha VM, Souza DB. A concepção sobre saúde do escolar entre professores do ensino fundamental (1⿳⺈ a $4^{\text {a }}$ séries). Rev Hist Cienc Saúde - Manguinhos [periódico na internet].2005 [acesso em 14 set. 2010];12(2):28391. Disponível em: http://www.scielo.br/scielo. php?pid=S0104-59702005000200004\&script=sci_arttext

\section{CONTRIBUIÇÃOS DOS AUTORES}

Pâmela Leites de Souza participou de todas as etapas da construção do artigo.Celeste dos Santos Pereira contribuiu como orientadora e revisora do trabalho. Maria Laura Silveira Nogueira, Denise Bermudez Pereira e Fabiana de Oliveira Möler foram organizadoras iniciais do projeto "Educacando para a saúde", tendo contribuído na construção da metodologia e resultados, além de atuarem como revisoras. Giolana Mascarenhas da Cunha auxiliou em todas as etapas e na formatação do trabalho.

\section{CONFLITO DE INTERESSES}

Declarou não haver.

\section{ENDEREÇO PARA CORRESPONDÊNCIA}

Pâmela Leites de Souza

Rua General Osório, n450/apt 202

Centro - Pelotas

CEP. 96020-000 RS 